and because they are sufficiently faint that they do not represent a major disorder in the structure, we have not investigated them further. It does seem likely that they represent such a limited disorder that no doubt remains about the correctness of the ordered structure.

We wish to thank Prof. W. E. Parham for supplying the sample of 1,4-dithiadiene used in this study. Financial support (to P. H.) by a Shell Oil Company Fellowship and (to R. C.) by a E. I. du Pont de Nemours Company Fellowship is gratefully acknowledged. A portion of this research was supported by ONR and OOR contracts.

\section{References}

Allen, P. W. \& Sutton, L. E. (1950). Acta Cryst. 3, 46. BEACH, J. Y. (1941). J. Chem. Phys. 9, 54.

Beevers, C. A. \& Robertson, J. H. (1950). Acta Cryst. 3, 164.
Donohue, J. \& Schomaker, V. (1948). J. Chem. Phys. 16, 92.

Karle, I. L. \& Karle, J. (1952). J. Chem. Phys. 20, 63.

Lappin, G. R. \& Summerbell, R. K. (1948). J. Organ. Chem. 13, 671 .

Levi, L. E. (1890). Chem. News, 62, 216.

Lipscomb, W. N. (1946). Ph.D. Thesis, California Institute of Technology.

Parham, W. E., Wynberg, H. \& Ramp, F. L. (1953). J. Amer. Chem. Soc. 75, 2065.

Pauling, L. (1942). The Nature of the Chemical Bond. Ithaca: Cornell University Press.

Pickett, L. W. \& Sheffield, E. (1946). J. Amer. Chem. Soc. 68, 216.

Reed, T. B. \& Lipscomb, W. N. (1953). Acta Cryst. 6, 45.

Robertson, J. M. (1953). Organic Crystals and Molecules. Ithaca: Cornell University Press.

Schomaker, V. \& Pauling, L. (1939). J. Amer. Chem. Soc. 61, 1769.

Stevenson, D. P. \& Beach, J. Y. (1938). „J. Amer. Chem. Soc. 60, 2872.

WASER, J. (1951). Rev. Sci. Instrum. 22, 567.

Acta C'ryst. (1954). 7, 503

\title{
The Effect of Anisotropic Thermal Vibration on the Atomic Scattering Factor
}

\author{
By W. CochraN \\ Crystallographic Laboratory, Cavendish Laboratory, Cambridge, England
}

(Received 11 March 1954)

\begin{abstract}
A discussion of the effect of anisotropic thermal vibration of the atoms in a crystal on the intensity of the Bragg reflexions leads to a method by which these anisotropic temperature factors can easily be evaluated numerically.
\end{abstract}

It is well known that the effect of isotropic thermal vibration of atoms in a crystal is to reduce the atomic scattering factor for Bragg reflexions by a factor

$$
\exp \left[-2 \pi^{2} u^{2} S^{2}\right]
$$

where $u^{2}$ is the mean square displacement of an atom from its average position and $S=2 \sin \theta / \lambda$. It has generally been assumed that when the thermal vibration is anisotropic, the surfaces of constant temperature factor in reciprocal space are ellipsoids. As far as I am aware, no proof of this has been offered. The following derivation shows the circumstances in which the result is true, and has the merit of suggesting a way in which the calculation of structure factors, with allowance for anisotropic temperature effects, can be simplified.

Let $r$, having rectangular components $(x, y, z)$, be a distance in space, and $S$, with rectangular components $(\xi, \eta, \zeta)$, a distance in reciprocal space. The directions $(x, y, z)$ and $(\xi, \eta, \zeta)$ coincide; they are not however the directions of the crystallographic axes (which need not be rectangular), but are the 'principal directions of vibration' of an atom. Let $\varrho_{0}(r)$ be the electron distribution in the atom at rest, and $f_{0}(S)$ its atomic scattering factor. $f_{0}(S)$ is the Fourier transform of $\varrho_{0}(r)$; the latter is assumed to have spherical symmetry and therefore $f_{0}(S)$ also has this symmetry. Now suppose the atom to be displaced from its mean position so that the probability that its centre lies within a volume element $d x d y d z$ is

$$
\begin{aligned}
& p(x, y, z) d x d y d z=\left((2 \pi)^{3 / 2} u_{x} u_{y} u_{z}\right)^{-1} \\
& \quad \times \exp \left[-\left(x^{2} / 2 u_{x}^{2}+y^{2} / 2 u_{y}^{2}+z^{2} / 2 u_{z}^{2}\right)\right] d x d y d z,
\end{aligned}
$$

$u_{x}^{2}, u_{y}^{2}$ and $u_{z}^{2}$ being the mean square displacements in the three directions at right angles. The resulting average electron distribution $\varrho(r)$ may be described as the convolute of $\varrho_{0}(r)$ with $p(x, y, z)$. It follows that the resulting atomic scattering factor, $f(S)$, is the product of $f_{0}(S)$ with the Fourier transform of $p(x, y, z)$. This transform may be shown to be 


$$
\exp \left[-2 \pi^{2}\left\{\xi^{2} u_{x}^{2}+\eta^{2} u_{y}^{2}+\zeta^{2} u_{z}^{2}\right\}\right]
$$

(For a fuller discussion of points raised in this paragraph, see, for example, James, 1954, Appendix V.) That is,

$$
f(\xi, \eta, \zeta)=f_{0}(S) \exp \left[-2 \pi^{2}\left\{\xi^{2} u_{x}^{2}+\eta^{2} u_{y}^{2}+\zeta^{2} u_{z}^{2}\right\}\right]
$$

This result shows that the surfaces of constant scattering factor are ellipsoids provided that the thermal vibration is specified by the distribution (2) and that $f_{0}(S)$ is isotropic. The assumption of a distribution of this form is reasonable, and is in line with the assumption made by Debye $(1913 a, b, c)$ for the case of isotropic vibration. The calculation of structure factors when a crystal contains even one atom per unit cell having an anisotropic temperature factor requires a great deal of numerical work. Much of this can be avoided in a way which will now be described.

A convenient graphical method of evaluating $\exp \left[-2 \pi^{2} u^{2} S^{2}\right]$ is based on the fact that for a particular reciprocal-lattice point the value of $S$ is just the distance of the point from the origin. The method will not be described here as it is an obvious modification of that used to evaluate graphically the Lorentz polarization factor (Cochran, 1948). It will now be shown that there exists another lattice, obtained by a homogeneous distortion of the original, which has the property that the distance $S^{\prime}$ of a point from the origin, when substituted in the isotropic factor (1) gives the anisotropic factor (3). The graphical method can therefore be used to evaluate (3) just as easily as to evaluate (1). This can be seen from the fact that if in the expression (1) we replace $\xi$ by $\xi u_{x} / u$ etc. we obtain (3). In other words, the isotropic factor for the point $\left(\xi u_{x} / u, \eta u_{y} / u, \zeta u_{z} / u\right)$ is just the anisotropic factor for the point $(\xi, \eta, \zeta)$. The distorted reciprocal lattice is therefore obtained by homogeneous distortion of the original reciprocal lattice by factors $u_{x} / u, u_{y} / u, u_{z} / u$ in the $x, y$ and $z$ directions respectively.

Let the direction cosines of $\mathrm{a}^{*}$ relative to $(x, y, z)$ be $l_{a^{*}}, m_{a^{*}}, n_{a^{*}}$ and similarly for $\mathbf{b}^{*}$ and $\mathbf{c}^{*}$. The lattice translations, and interaxial angles, of the distorted reciprocal lattice are then given by

$$
\left(a^{*}\right)^{1}=\left(a^{*} / u\right)\left(l_{a^{*}}^{2} u_{x}^{2}+m_{a^{*}}^{2} u_{y}^{2}+n_{a^{*}}^{2} u_{z}^{2}\right)^{\frac{1}{2}} \text { etc., }
$$

and $\cos \left(\alpha^{*}\right)^{1}$

$$
=\frac{u_{x}^{2} l_{b^{\star}} l_{c^{\star}}+u_{y}^{2} m_{b^{\star}} m_{c^{\star}}+u_{z}^{2} n_{b^{\star}} n_{c^{*}}}{\left(u_{x}^{2} l_{b^{\star}}^{2}+u_{y}^{2} m_{b^{*}}^{2}+u_{z}^{2} n_{b^{\star}}^{2}\right)^{\frac{1}{2}}\left(u_{x^{*}}^{2} l_{c^{\star}}^{2}+u_{y}^{2} m_{c^{\star}}^{2}+u_{z}^{2} n_{c^{\star}}^{2}\right)^{\frac{1}{2}}} \text { etc. }
$$

It will in many instances, however, be easier to specify the direction cosines of $\mathbf{a}, \mathbf{b}$ and $\mathbf{c}$ in the system $(x, y, z)$. Let those of a be $l_{a}, m_{a}, n_{a}$.

Then there exists a distorted lattice in direct (crystal) space given by

$$
\begin{gathered}
a^{1}=a u\left(l_{a}^{2} / u_{x}^{2}+m_{a}^{2} / u_{y}^{2}+n_{a}^{2} / u_{z}^{2}\right)^{\frac{1}{2}}, \text { etc., } \\
\cos \alpha^{1} \quad \frac{\left(l_{b} l_{c} / u_{x}^{2}+m_{b} m_{c} / u_{y}^{2}+n_{b} n_{c} / u_{z}^{2}\right)^{\frac{1}{2}}}{\left(l_{b}^{2} / u_{x}^{2}+m_{b}^{2} / u_{y}^{2}+n_{b}^{2} / u_{z}^{2}\right)^{\frac{1}{2}}\left(l_{c}^{2} / u_{x}^{2}+m_{c}^{2} / u_{y}^{2}+n_{c}^{2} / u_{z}^{2}\right)^{\frac{1}{2}}} \text { etc. }
\end{gathered}
$$

whose reciprocal lattice also defines the required lattice $\left(a^{*}\right)^{\mathbf{1}},\left(b^{*}\right)^{1},\left(c^{*}\right)^{\mathbf{1}}$. As found in this way, two stages of calculation are therefore required, but the calculation begins from quantities which are more easily determined.

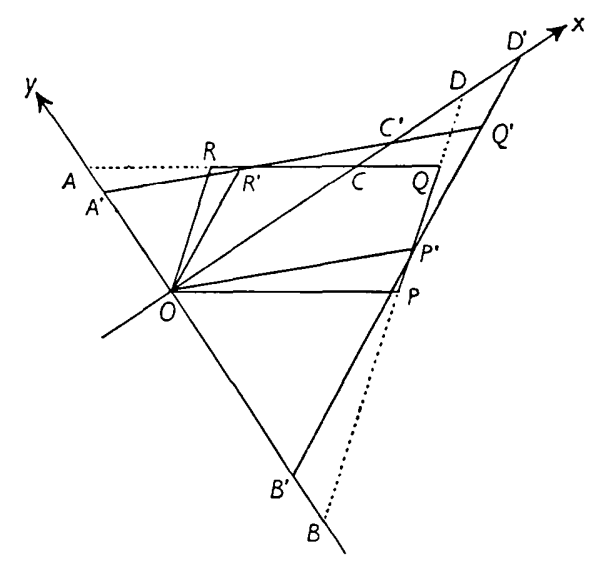

Fig. 1. Graphical construction for the distorted reciprocal lattice in two dimensions. The parallelogram $O P Q R$ defines the reciprocal lattice, $x$ and $y$ are directions of maximum and of minimum thermal vibration respectively. It is assumed that $u_{x} / u=1.2$ and $u_{y} / u=0.8$. The points $A^{\prime}$ and $B^{\prime}$ on the $y$ axis are such that $O A^{\prime}\left|O A=O B^{\prime}\right| O B=0 \cdot 8$, while $C^{\prime}$ and $D^{\prime}$ on the $x$ axis are such that $O C^{\prime}\left|O C=O D^{\prime}\right| O D=$ 1.2. The defining vectors of the distorted reciprocal lattice are therefore parallel to $A^{\prime} C^{\prime}$ and $B^{\prime} D^{\prime}$ respectively, so that the parallelogram $O P^{\prime} Q^{\prime} R^{\prime}$ gives one cell of the distorted reciprocal lattice.

From the cumbersome nature of the formulae set out above it may be wondered what has become of the simplicity claimed for the method. Usually, however, the symmetry of the lattice is higher than triclinic, and further simplifications ensue if one of the directions $(x, y, z)$ coincides with a crystallographic axis. In dealing with structure factors belonging to one zero layer of the reciprocal lattice, the distorted reciprocal lattice can be obtained from the original by a simple graphical construction shown in Fig. 1.

\section{References}

Cochran, W. (1948). J. Sci. Instrum. 25, 253.

Debye, P. (1913a). Verh. dtsch. phys. Ges. 15, 678.

Debye, P. (1913b). Verh. dtsch. phys. Ges. 15, 738.

DeBye, P. (1913c). Verh. dtsch. phys. Ges. 15, 857.

JAMES, R. W. (1954). The Crystalline State, vol. 2, 2nd ed. London: Bell. 